\title{
The B3-VLA CSS sample
}

\section{VLBA images at 6 and $3.6 \mathrm{~cm}^{\star, \star \star}$}

\author{
M. Orienti ${ }^{1,2}$, D. Dallacasa ${ }^{1,2}$, C. Fanti ${ }^{2,3}$, R. Fanti ${ }^{2,3}$, S. Tinti ${ }^{4}$, and C. Stanghellini ${ }^{5}$ \\ 1 Dipartimento di Astronomia, Università di Bologna, via Ranzani 1, 40127 Bologna, Italy \\ e-mail: orienti@ira.cnr.it \\ 2 Ist. di Radioastronomia - CNR, via Gobetti 101, 40129 Bologna, Italy \\ 3 Dipartimento di Fisica, Università di Bologna, via Irnerio 46, 40126 Bologna, Italy \\ 4 SISSA/ISAS, Via Beirut 4, 34014 Trieste, Italy \\ 5 Ist. di Radioastronomia - CNR, CP 141, 96017 Noto (SR), Italy
}

Received 30 April 2004 / Accepted 6 July 2004

\begin{abstract}
We present the results of VLBA observations at 6 and $3.6 \mathrm{~cm}$ for eighteen Compact Steep Spectrum radio sources (CSS) from the B3-VLA CSS sample. In most cases these VLBA images display a "Double/Triple" morphology, and the core is unambiguously detected in seven sources. Multifrequency images allow us to study the spectral properties of the individual source components and to constrain the spectral age. In lobe-dominated sources the radiative ages deduced from the synchrotron theory are in the range of up $5 \times 10^{3}$ years, if equipartition magnetic field is assumed. Polarized emission is detected at a few percent level for two sources only. They were the only two sources displaying polarized emission in VLA A configuration data, and this implies that beam depolarization is not effective in reducing the integrated fractional polarization for these sources.
\end{abstract}

Key words. galaxies: active - galaxies: nuclei - radio continuum: galaxies - galaxies: quasars: general - polarization

\section{Introduction}

Compact Steep-Spectrum (CSS) and the GHz PeakedSpectrum (GPS) radio sources are powerful $\left(P_{1.4 \mathrm{GHz}}>\right.$ $10^{25} \mathrm{~W} / \mathrm{Hz}$ ) extragalactic objects characterized by a small physical size $\left(\leq 20 \mathrm{~h}^{-1} \mathrm{kpc}^{1}\right)$. They represent a significant fraction $(\simeq 15-30 \%)$ of the radio source population at $5 \mathrm{GHz}$, and are associated with distant objects $(z>0.2)$, both galaxies and quasars.

It is currently accepted that at least those with twosided radio emission represent the young stage in the radio source evolution, from Compact (size $<1 h^{-1} \mathrm{kpc}$ ) to Large (size $>20 h^{-1} \mathrm{kpc}$ ) Symmetric Objects (Fanti et al. 1995; Readhead et al. 1996; Snellen et al. 2000, 2003). Strong support to this scenario comes from the measurements of proper motions of the hot spots of Compact Symmetric Objects (CSO), (see the review by Polatidis \& Conway 2003), with separation velocities of $\sim 0.1-0.4 h^{-1} \mathrm{c}$, and then kinematic ages less than

* Figures 1-3 are only available in electronic form at http://www . edpsciences.org

$\star \star$ Tables 1 and 2 are only available in electronic form at the CDS via anonymous ftp to cdsarc.u-strasbg.fr $(130.79 .128 .5)$ or via http://cdsweb.u-strasbg.fr/cgi-bin/gcat?]/A+A/426/463

1 We assume $H_{0}=100 h \mathrm{~km} \mathrm{~s}^{-1} \mathrm{Mpc}^{-1}, q_{0}=0.5$, as in Papers I-III.
$3 \times 10^{3}$ years for the smallest objects. Moreover, the spectral ages based on synchrotron ageing and derived from total flux density measurements, are about $10^{3}-10^{4}$ years, in agreement with kinematic ages (Murgia et al. 1999; Murgia 2003).

Recently, we have selected a new complete sample of 87 CSSs (size $<20 h^{-1} \mathrm{kpc}$; Fanti et al. 2001) from the B3-VLA catalogue (Vigotti et al. 1989). VLA A-configuration observations at $1.4,4.9$ and $8.4 \mathrm{GHz}$ allowed a correct morphological classification for about half of the sources (Paper I). However, eighteen objects were only marginally resolved at $8.4 \mathrm{GHz}$ (beam size $\sim 0.2^{\prime \prime}$ ), and twenty-eight were unresolved. All these were then observed at $1.67 \mathrm{GHz}$ with VLBI: the former group was investigated by means of the joint EVN and MERLIN (Dallacasa et al. 2002b, Paper III), while the latter set was observed with the VLBA (Dallacasa et al. 2002a, Paper II).

This paper reports on the results of new VLBA plus a single VLA antenna observations at higher frequencies of the most compact objects among those studied in Paper II. Based on the sizes and flux densities of the components detected at $1.67 \mathrm{GHz}$, ten sources were observed at both 5.0 and $8.4 \mathrm{GHz}$, and eight at $5.0 \mathrm{GHz}$ only.

Spectral index distribution and solid morphological classification of source components, along with polarization 
information, will be presented in this work.

The layout of this paper is the following:

- Sect. 2 describes the new VLBA observations;

- Sect. 3 reports on data reduction;

- images of the source total intensity and spectral index distri-

bution are shown and discussed in Sect. 4;

- notes on each source are in Sect. 5;

- a concise discussion and a brief summary are given in Sects. 6 and 7.

\section{The source list and the VLBA observations}

Among the 28 sources with VLBA observations at $1.67 \mathrm{GHz}$ (Paper II), we included in the new observing run only those for which our estimate of the peak brightness at 5.0 and $8.4 \mathrm{GHz}$ was high enough to carry out standard observations, without the need of phase referencing. A short summary of the properties of the sources investigated in this paper is given in Table 1 .

The VLBA plus a single VLA antenna observations were carried out on November 5, 2001 in full polarization mode recording at $64 \mathrm{Mbps}$, for a total of $24 \mathrm{~h}$. Each source was observed for a total of about $30 \mathrm{~min}$ at each frequency. A single VLA antenna was added to the VLBA in order to provide a good set of relatively short baselines. Since the sources are relatively strong and have quite small angular sizes, snap-shot observing mode was considered adequate, although it is known that this may cause some flux density losses particularly for the fainter and more extended source components (Sect. 3).

\section{Data reduction}

After the application of system temperature and antenna gain information, the amplitudes were checked by means of the data on DA193 (J0555+398) which is unresolved on a large number of baselines. All the gain corrections were found to be within $5 \%$ which can be conservatively assumed as absolute calibration error.

The instrumental polarization has been removed by using the data on the unpolarized source OQ208, while the absolute position of the electric vector has been determined by the combination of data for DA193 and 3C 279 as compared to the VLA/VLBA polarization calibration database. The corrections derived from the two sources were in good agreement $\left(\leq 2^{\circ}\right)$.

The final radio images were obtained after a number of phase self-calibration iterations by means of the NRAO AIPS package.

The final rms noise $(1 \sigma)$ is usually in the range $\sim 0.1-0.18 \mathrm{mJy} /$ beam and $\sim 0.08-0.15 \mathrm{mJy} /$ beam at 5.0 and $8.4 \mathrm{GHz}$ respectively, in agreement with the expected thermal noise. The actual noise in each image (Figs. 1 and 2) can be estimated from the first contour $(3 \sigma)$. The dynamic range, defined as the ratio of peak brightness to $1 \sigma$, ranges from 200 to 1300 at both frequencies. The typical full resolution is $\sim 2 \times 3$ mas at $5.0 \mathrm{GHz}$ and $\sim 1.5 \times 2$ mas at $8.4 \mathrm{GHz}$.

The total flux density of each source has been measured by using the AIPS verb TVSTAT. The brightest and most compact sub-structures of each source have been fitted to an elliptical Gaussian model by means of the AIPS task JMFIT. The Full Width Half Maximum ( $F W H M$ ) obtained in this way are reported in Table 2. For more extended sub-components, the flux density has been measured by using TVSTAT. In this case, the angular size, measured from the lowest reliable contours, is roughly twice the size of the FWHM of a conventional Gaussian covering a similar area.

Sub-components are referred to as North (N), South $(\mathrm{S})$, East (E), West (W), Central (Ce), Jet (J) and flat spectrum Core (C) when detected, following the labelling already used in Paper II.

The parameters of sub-components are given in Table 2, whose layout is described in the caption.

There is clearly a systematic flux density deficiency, typically $\sim 10 \%$, in the images at both 5.0 (we have taken into account a $\sim 3 \%$ decrement from the $4.86 \mathrm{GHz}$ VLA flux density) and $8.4 \mathrm{GHz}$, as compared to the values derived from VLA images (Paper I). Also in the VLBA observations at $1.67 \mathrm{GHz}$ there was a systematic flux density deficiency with respect to the one extrapolated from the $1.4 \mathrm{GHz}$ VLA measure (typically $\geq 5 \%$, Paper II).

The snap-shot observing mode may be not effective in the detection of relatively large components covering a substantial number of pixels and this effect gets worse with frequency given that the short spacings sampling becomes sparser.

\section{Source images}

\subsection{Total intensity and polarization}

Full resolution images of the 10 sources with both 5.0 and $8.4 \mathrm{GHz}$ data are presented, next to each other, in Fig. 1. Figure 2 shows images of the sources with $5.0 \mathrm{GHz}$ data only.

Besides the total intensity $(I)$, images in the Stokes' $U$ and $Q$ parameters at both 5.0 and $8.4 \mathrm{GHz}$ were produced with the final fully calibrated dataset. Typical noise levels in these images are about $0.07-0.10 \mathrm{mJy} /$ beam for all sources. Polarization intensity images were corrected for the positive bias.

Only B3-1343+386 and B3-2304+377 did show some amount of polarized emission and their polarization properties are discussed in Sect. 6.3.

\subsection{Spectral index images}

For each source, besides the full-resolution images, we also produced low-resolution images at both frequencies 5.0 and $8.4 \mathrm{GHz}$, using the same $u v$-ranges, image sampling and restoring beams of the $1.67 \mathrm{GHz}$ already available (Paper II). All these new images were obtained with natural grid weighting in order to make the differences in the sampling density at short spacing less effective.

In Fig. 3 we show for all sources the greyscale spectral index images between 1.67 and $5.0 \mathrm{GHz}$ superimposed on the $5 \mathrm{GHz}$ contours obtained from the low-resolution images. Image registration (LGEOM) was carried out by comparing the location of optically thin bright features. This led to satisfactory 
results, generally without any significant gradient in the direction transverse to the source axis.

For the 10 sources observed at 1.67, 5.0 and $8.4 \mathrm{GHz}$, we produced also multi-frequency spectra of each source component: two examples are shown in Fig. 6, and general results will be briefly outlined in Sect. 6.2.

\section{Notes on individual sources}

In this section we discuss each source in the light of Figs. 1-3, which allow to draw a fair morphological information. Most sources are associated with either galaxies or empty fields; the two quasars are remarked in the discussion on individual objects.

We note that the spectral index values reported in the following discussion have to be considered as local spectral indices, in order to point out the presence of a possible hot spot. For this reason these values may appear different from those reported in Table 2, which, instead, have been computed by averaging the total flux density of the whole component at each frequency.

\subsection{Sources observed at both 5.0 and $8.4 \mathrm{GHz}$}

- B3-0147+400: This source is a Triple with a clear detection of the core (component $\mathrm{C}$ ) located at the Westernmost edge of the most luminous lobe. It was blended into $\mathrm{E}$ component at $18 \mathrm{~cm}$ (Paper II). At $8.4 \mathrm{GHz}$ the core (accounting for $18.5 \mathrm{mJy}$, i.e. $10 \%$ of the total flux density as measured at the VLA) is further resolved, with the presence of a few mJy jetlike emission. The core spectral index is inverted, with a local value of $\alpha_{5.0}^{8.4} \sim-0.5$ slightly more inverted than $\alpha_{1.7}^{5.0} \sim-0.4$ seen in Fig. 3 (see also Table 2). The radio spectrum of the "core region", plotted in Fig. 6, is seen with a lower resolution and then all the spectral index measures reported in the figure also include a substantial contribution from the steep spectrum lobe emission seen at $18 \mathrm{~cm}$ (Paper II). The source structure is very asymmetric: the core is located at $\sim 10$ and $\sim 55$ mas from the edges of the Eastern and Western components respectively. The flux density ratio between the two lobes is $S_{\mathrm{E}} / S_{\mathrm{W}} \sim 2.1$ at $5.0 \mathrm{GHz}$ and $\sim 5.2$ at $8.4 \mathrm{GHz}$, where most of the diffuse emission in the $\mathrm{W}$ lobe is resolved out. About $20 \%$ and $15 \%$ of the total flux density is missing in our VLBA images at 5.0 and $8.4 \mathrm{GHz}$ respectively. We note that the diffuse component $\mathrm{T}^{*}$ in Paper II, accounts for $113 \mathrm{mJy}$ at $18 \mathrm{~cm}$ and it is completely resolved out at 6 and $3.6 \mathrm{~cm}$. With a spectral index of 1.0 it would account for 41 and $22 \mathrm{mJy}$ at 6 and $3.6 \mathrm{~cm}$, reducing the missing flux density to $9 \%$ at $5.0 \mathrm{GHz}$ and to $5 \%$ at $8.4 \mathrm{GHz}$. This consideration may also apply to other sources where the more extended components at $18 \mathrm{~cm}$ are only partially accounted for at higher frequencies.

- B3-0703+468: This source appears as a Double. The radioemission originates within two well-resolved components, $\sim 50$ mas apart. Their flux density ratio is $S_{\mathrm{W}} / S_{\mathrm{E}} \sim 1.6$ at both 5.0 and $8.4 \mathrm{GHz}$. At the resolution of the $18 \mathrm{~cm}$ data $\left(7.55 \times 4.91 \mathrm{mas}\right.$ in PA $\left.13^{\circ}\right)$ the central regions of the two components, probably the hot spots, have a local spectral index $\alpha_{1.7}^{5.0} \sim 0.6$, which is flatter than the outer parts of the lobes (Fig. 3). About $15 \%$ of the total flux density is missing in both VLBA images at 5.0 and $8.4 \mathrm{GHz}$. The core is not detected in between the two lobes.

- B3-0822+394: At full resolution this source appears rather fragmented, mostly due to the not adequate sampling of the short $u v$ spacings. It was undetected on the longest baselines, and therefore the restoring beam of the image in Fig. 1 is larger than for other sources. It may be classified as a Double with a total extent of $\sim 60$ mas $(L L S \sim 260 \mathrm{pc})$. The flux density ratio is $S_{\mathrm{E}} / S_{\mathrm{W}} \sim 3.6$ at $5.0 \mathrm{GHz}$ and $\sim 4.7$ at $8.4 \mathrm{GHz}$. Both components have a steep spectrum $\left(\alpha_{1.7}^{5.0} \geq 1\right.$; Table 2$)$. About $15 \%$ and $25 \%$ of the total flux density is missing in our VLBA images at 5.0 and $8.4 \mathrm{GHz}$ respectively. The core is not detected.

- B3-0840+424: The images presented in Fig. 1, undoubtedly label this source as a Triple, with a clear detection of the core, embedded in the Southernmost edge of the most luminous lobe. At $8.4 \mathrm{GHz}$ the core region (accounting for $31.2 \mathrm{mJy}$, i.e. $8 \%$ of the source total flux density as measured at the VLA) is further resolved, with the presence of a few mJy jet-like feature pointing to the South. The core spectral index is inverted, $\alpha_{5.0}^{8.4} \sim-0.03$ (Table 2). The structure of this source is very asymmetric: the core is located at $\sim 15$ and $\sim 100$ mas from the edges of the Northern and Southern components respectively. The flux density ratio between the two lobes is $S_{\mathrm{N}} / S_{\mathrm{S}} \sim 6.0$ at $5.0 \mathrm{GHz}$ and $\sim 7.1$ at $8.4 \mathrm{GHz}$. At the resolution of the $1.67 \mathrm{GHz}$ data $\left(7.56 \times 5.29\right.$ mas in PA $\left.15.56^{\circ}\right)$, the brightest region of the Northern component, probably dominated by a hot spot, has a local spectral index $\alpha_{1.7}^{5.0} \sim 0.5$, while the Southern one has a steeper spectrum $\left(\alpha_{1.7}^{5.0} \sim 0.8\right.$ but 0.5 on the side nearest to the core and 1.1 on the other; Fig. 3). About $15 \%$ of the total flux density is missing in both VLBA images at 5.0 and $8.4 \mathrm{GHz}$.

- B3-1133+432: This source appears as a Double. The radio emission originates mainly within two well-resolved components, with a flux density ratio $S_{\mathrm{N}} / S_{\mathrm{S}} \sim 2.0$ at both 5.0 and $8.4 \mathrm{GHz}$. Their brightest regions, probably hot spots, have the same local spectral index $\alpha_{1.7}^{5.0} \sim 0.8$ (Fig. 3), while the total spectral indices are steeper (Table 2). The core is not detected. About $14 \%$ and $17 \%$ of the total flux density is missing in our VLBA images at 5.0 and $8.4 \mathrm{GHz}$ respectively.

- B3-1242+410: This is one of the two sources identified with a quasar. The morphology of this source is quite similar to the source $1946+708$, classified as a Compact Symmetric Object (CSO) by Peck \& Taylor (2001). The radio structure is dominated by the core and a straight one-sided jet, although substantial emission is present on the counter jet side. The core (component $\mathrm{C}$ ) accounts for $46.0 \mathrm{mJy}$ at $8.4 \mathrm{GHz}$, i.e. $10 \%$ of the source total flux density measured at the VLA. Its spectral index is $\alpha_{5.0}^{8.4} \sim-0.32$ (Table 2).

About $20 \%$ of the total flux density is missing in both VLBA images at 5.0 and $8.4 \mathrm{GHz}$. This is mostly due to the extended 
features surrounding the Southern component which are much too resolved by our high frequency observations, as proved by comparison of the VLBA image at $1.67 \mathrm{GHz}$ (2002, Paper II). The rms noise in the image plane is somehow higher than in other sources possibly due to the presence of unconstrained flux density.

- B3-1343+386: This is the other source identified with a quasar. A weak feature, visible only in VLBA images at $1.67 \mathrm{GHz}$ (Paper II), is present to the North of the Northern component visible on the images in Fig. 1, so the radio morphology is that of a Triple. The sub-structure labelled as component $\mathrm{C}$ in Fig. 1, is the source core; in fact it has a flat spectrum (in agreement with Marecki et al. 2003), with a local spectral index $\alpha_{5.0}^{8.4} \sim 0.1$ (Fig. 3), while the spectral index of the whole component is steeper $(\sim 0.7$, see Table 2$)$ since substantial jet emission enters in the latter measurement. At $8.4 \mathrm{GHz}$ the core region accounts for $50.3 \mathrm{mJy}$, i.e. $18 \%$ of the source total flux density as measured at the VLA. This source is one of the two objects with a significant polarization emission (Sect. 6.3). Since the extended emission between the core region and the Southern component is almost completely lost in high frequency images, about $15 \%$ and $30 \%$ of the total flux density is missing in our VLBA images at 5.0 and 8.4 GHz respectively. Also for this source the rms noise in the image plane is somehow higher than the average value possibly due to emission nearly completely resolved out by the VLBA observations.

- B3-1432+428: This source may be classified as a Double. The weak feature, visible in VLBA images at $1.67 \mathrm{GHz}$ (2002, Paper II), is not detected in these observations. At the resolution of the $18 \mathrm{~cm}$ data $\left(6.36 \times 5.92\right.$ mas in $\left.\mathrm{PA} 1.77^{\circ}\right)$, the brightest region of the Eastern component, probably a hot spot, has a local spectral index $\alpha_{1.7}^{5.0} \sim 0.4$ (Fig. 3). The Western lobe has $\alpha_{1.7}^{5.0} \sim 1.2$ (Table 2). The core is not detected. About $20 \%$ of the total flux density is missing in both VLBA images at 5.0 and $8.4 \mathrm{GHz}$.

- B3-2330+402: The images presented in Fig. 1 suggest that this source is an asymmetric Triple. At the resolution of the $1.67 \mathrm{GHz}$ data $\left(8.05 \times 4.29\right.$ mas in $\left.\mathrm{PA}-12.09^{\circ}\right)$ the Western component, which is the most luminous one, has a total spectral index $\alpha_{1.7}^{5.0} \sim 0.4$, while all the other structures have steeper spectra (Table 2). This component is resolved into a relatively compact emission located at the outer edge of some diffuse emission extending towards the remaining parts of the source. The morphology is reminiscent that of $2342+821$ (Dallacasa et al. 1995). The core is not detected. About $20 \%$ and $10 \%$ of the total flux density is missing in our VLBA images at 5.0 and $8.4 \mathrm{GHz}$.

- B3-2358+406: The radio structure of this source is quite complex. The source core (component C) accounts for 21.4 and $23.6 \mathrm{mJy}$ at 5.0 and $8.4 \mathrm{GHz}$ respectively, i.e. $3 \%$ and $6 \%$ of the source total flux density as measured at the VLA, with the presence of a few mJy jet-like emission. The spectral index of the core region is inverted, $\alpha_{5.0}^{8.4} \sim-0.2$ (Table 2). The flux density ratio of the two components identified with lobes is $S_{\mathrm{S}} / S_{\mathrm{N}} \sim 4.5$ and $\sim 5.6$ at 5.0 and $8.4 \mathrm{GHz}$ respectively, and at the resolution of the $18 \mathrm{~cm}$ data $(8.07 \times 4.22$ mas in PA $-11.12^{\circ}$ ), there are two brighter regions, probably hot spots, whose local spectral indices are flatter than those of the outer lobes (Fig. 3). About $16 \%$ and $18 \%$ of the total flux density is missing in our VLBA images at 5.0 and $8.4 \mathrm{GHz}$ respectively.

\subsection{Sources observed at $5.0 \mathrm{GHz}$ only}

- B3-0039+373: This source is a relatively symmetric Double, with a flux density ratio $S_{\mathrm{N}} / S_{\mathrm{S}} \sim 1.1$. The brighter regions of the two components have a local spectral index $\alpha_{1.7}^{5.0} \sim 0.8$ (Fig. 3) around their peak brightness, possibly hot spots with substantial contamination from steep spectrum lobe emission. The spectral index gets substantially steeper in the backflow tails of the lobes. A core candidate could not be detected. About $15 \%$ of the total flux density is missing in our VLBA image at $5.0 \mathrm{GHz}$.

- B3-1008+423: The radio structure of this source is asymmetric and rather complex, with a flux density ratio $S_{\mathrm{W}} / S_{\mathrm{E}} \sim 7.7$. The central region is probably a weak jet. The bright region in the Western component, likely a hot spot, has a local spectral index $\alpha_{1.7}^{5.0} \sim 0.6$ (Fig. 3). The other components have steep spectra (Table 2). A core candidate could not be detected. About $20 \%$ of the total flux density is missing in our VLBA image at 5.0 GHz.

- B3-1049+384: It is not possible to unambiguously classify this complex and very asymmetric object. The Western components seem to be an independent very Compact Double, with an overall extent of $\sim 30$ mas ( $L L S \sim 140 \mathrm{pc}$ ) and a flux density ratio of the two components $S_{\mathrm{W} 2} / S_{\mathrm{W} 1} \sim 1.1$. All components have steep spectra, except for the central one (Component E1) which has a spectral index $\alpha_{1.7}^{5.0} \sim 0.3$, although this value is rather uncertain. About $18 \%$ of the total flux density is missing in our VLBA image at $5.0 \mathrm{GHz}$.

- B3-1136+383: This source is an asymmetric Triple with components well aligned in N-S. The sub-structure labelled as component $\mathrm{C}$ (Fig. 2) is the source core (accounting for $10.8 \mathrm{mJy}$ at $5.0 \mathrm{GHz}$, i.e. $5 \%$ of the source total flux density as measured at the VLA); in fact it has a flat spectrum with a local spectral index $\alpha_{1.7}^{5.0} \sim 0.1$ (Fig. 3), while the total spectral index of the whole component is steeper (Table 2) since it is affected by the presence of substantial jet emission. The Northern and the Southern components are respectively $\sim 35$ and $\sim 20$ mas far from the core. In these components, which have a flux density ratio $S_{\mathrm{N}} / S_{\mathrm{S}} \sim 2.7$, there are two brighter regions, probably hot spots, whose local spectral indices are lower $\left(\alpha_{1.7}^{5.0} \sim 0.5\right.$ for the component $\mathrm{N}$ and $\sim 0.6$ for the S, Fig. 3) than those of the lobe outer parts (Table 2). About $15 \%$ of the total flux density is missing in our VLBA image at $5.0 \mathrm{GHz}$. 
Table 3. Lobe spectral ages and estimated source expansion velocity; $v_{\mathrm{b}}$ and $B_{\mathrm{eq}}$ are the break frequency and the equipartition magnetic field. When redshift is unknown, $L L S$ is calculated assuming $z=1.00$, and the value is given with a leading " ".

\begin{tabular}{crrrrr}
\hline \hline Name & $\begin{array}{r}v_{\mathrm{b}} \\
\mathrm{GHz}\end{array}$ & $\begin{array}{r}B_{\text {eq }} \\
\mathrm{mG}\end{array}$ & $\begin{array}{c}t_{\text {syn }} \\
10^{3} \mathrm{yr}\end{array}$ & $\begin{array}{r}L L S \\
\mathrm{pc}\end{array}$ & $\begin{array}{c}v_{\text {sep }} \\
h^{-1} \mathrm{c}\end{array}$ \\
\hline & & & & & \\
$0147+400$ & 7.2 & 6.2 & 1.2 & $\sim 325$ & 0.44 \\
$0822+394$ & 22.0 & 3.3 & 1.8 & 260 & 0.24 \\
$0840+424$ & 24.0 & 2.7 & 2.3 & $\sim 530$ & 0.38 \\
$1242+410$ & 10.0 & 5.4 & 1.3 & 175 & 0.22 \\
$1432+428$ & 6.5 & 2.6 & 4.9 & $\sim 170$ & 0.06 \\
$2330+402$ & 5.3 & 4.0 & 2.9 & $\sim 365$ & 0.21 \\
$2358+406$ & 12.0 & 4.6 & 1.5 & $\sim 370$ & 0.40 \\
& & & & & \\
\hline
\end{tabular}

- B3-1159+395: This object can be classified as a Double. In the Northern and Southern components, whose flux density ratio is $S_{\mathrm{N}} / S_{\mathrm{S}} \sim 2.1$, there are two brighter regions, probably hot spots: their local spectral indices are $\alpha_{1.7}^{5.0} \sim 0.5$ and $\sim 0.6$ respectively (Fig. 3), flatter than those of the lobe outskirts (Table 2). The inner tail-like emission in the Northern component could be a jet, although its quite steep spectral index is more consistent with a backflow tail, commonly seen in radio lobes. A core candidate has not been found.

About $17 \%$ of the total flux density is missing in our VLBA image at $5.0 \mathrm{GHz}$.

- B3-1441+409: This source is a Triple with well-aligned components in NE-SW. The image presented in Fig. 2 clearly detects the core (component C), which accounts for a $29.9 \mathrm{mJy}$ emission, i.e. $8 \%$ of the source total flux density as measured at the VLA. The structure is asymmetric: a single bright jet emerges from the Western part of the core region, which is located at $\sim 40$ and $\sim 65$ mas far from the Eastern and Western components respectively. Their flux density ratio is $S_{\mathrm{W}} / S_{\mathrm{E}} \sim 4.1$. About $20 \%$ of the total flux density is missing in our VLBA image at $5.0 \mathrm{GHz}$.

- B3-2304+377: The radio morphology of this source appears rather complex and with a distorted structure. The brightest region of the Western component, probably a hot spot, has a local spectral index $\alpha_{1.7}^{5.0} \sim 0.4$ (Fig. 3), while the outer lobe and the other structures have much steeper spectra (Table 2). Right around the brightest region of this component, some significant amount of polarized emission is detected (Sect. 6.3). The Eastern part of this radio source is instead more complex, and more difficult to interpret. Component E1 turned out to have $\alpha_{1.7}^{5.0} \sim 0.2$. We note that the value reported as E1 total flux density in Paper II is wrong given that it refers to the whole East complex. The correct flux density for E1 at $1.67 \mathrm{GHz}$ is $54 \mathrm{mJy}$. Then E1 may be either the source core or a knot in the jet with substantial self absorption or, finally a hot spot. Components E2 and E3 have steep spectra, although this is also in part some amount of relatively extended emission resolved out by the present observations. In fact about $20 \%$ of the total flux density is missing in our VLBA image at $5.0 \mathrm{GHz}$.

- B3-2348+450: At full resolution this source is almost completely resolved out. The steep spectrum emission to the South of the Southern component is heavily resolved by the present observations. The source can be classified as a Double and the flux density ratio of the two components is $S_{\mathrm{S}} / S_{\mathrm{N}} \sim 2.5$. The core is not detected. About $18 \%$ of the total flux density is missing in our VLBA image at $5.0 \mathrm{GHz}$.

\section{Discussion}

\subsection{Morphology, cores and asymmetries}

The following discussion of the radio morphologies is based, in addition to the present data, on the $1.67 \mathrm{GHz}$ VLBA images presented in Paper II.

The majority of the sources shows a "Double/Triple" structure and the radio emission is dominated by extended components that can be interpreted in terms of radio lobes. In seven sources (the two quasars B3-1242+410 and B3-1343+386 and the five empty fields B3-0147+400, B3-0840+424, B3-1136+383, B3-1441+409, B3-2358+406) we unambiguously identified the core. Another one (B3-2304+377) has a tentative core identification.

The extended emission is distributed on either side of the core, although it may show large asymmetries both in arm ratio and in brightness (see B3-0147+400 and B3-0840+424). According to the definition by Readhead et al. (1996) these sources are firmly classified as CSOs. Four sources, all with detected cores (B3-1242+410, B3-1343+386, B3-1441+409 and B3-2358+406) show clearly the presence of luminous jets. In two of them, B3-1343+386 (quasar) and B3-1441+409 (empty field) the jet is one sided. In B3-1242+410 (quasar) we see a main jet very long and bright and a shorter but equally bright counterjet. Finally, in B3-2358+406 (empty field) we find a twin jet. In a few other sources (see Sect. 5) there are hints for the presence of faint jets.

The radio morphology of the lobes is reminiscent of the FRI/FRII morphology. Five sources with core (B3-0147+400, B3-0840+424, B3-1136+383, B3-1343+386 and B3-1441+409) appear of FRII type or intermediate between FRI and FRII, as they show a bright flatter spectrum compact feature in one or both lobes, which recall the hot spots in the powerful large size double sources. The remaining two objects (B3-1242+410 and B3-2358+406) appear of FRI type, as their radio emission is more bright in the inner regions as due to the jet contribution.

Among the sources without a core detection, six (B3-0039+373, B3-0703+468, B3-0822+394, B3-1008+423, $\mathrm{B} 3-1133+432$ and $\mathrm{B} 3-1159+395)$ are FRII or intermediate type and we assume that they are CSOs. The last four (B3-1049+384, B3-1432+428, B3-2330+402 and 

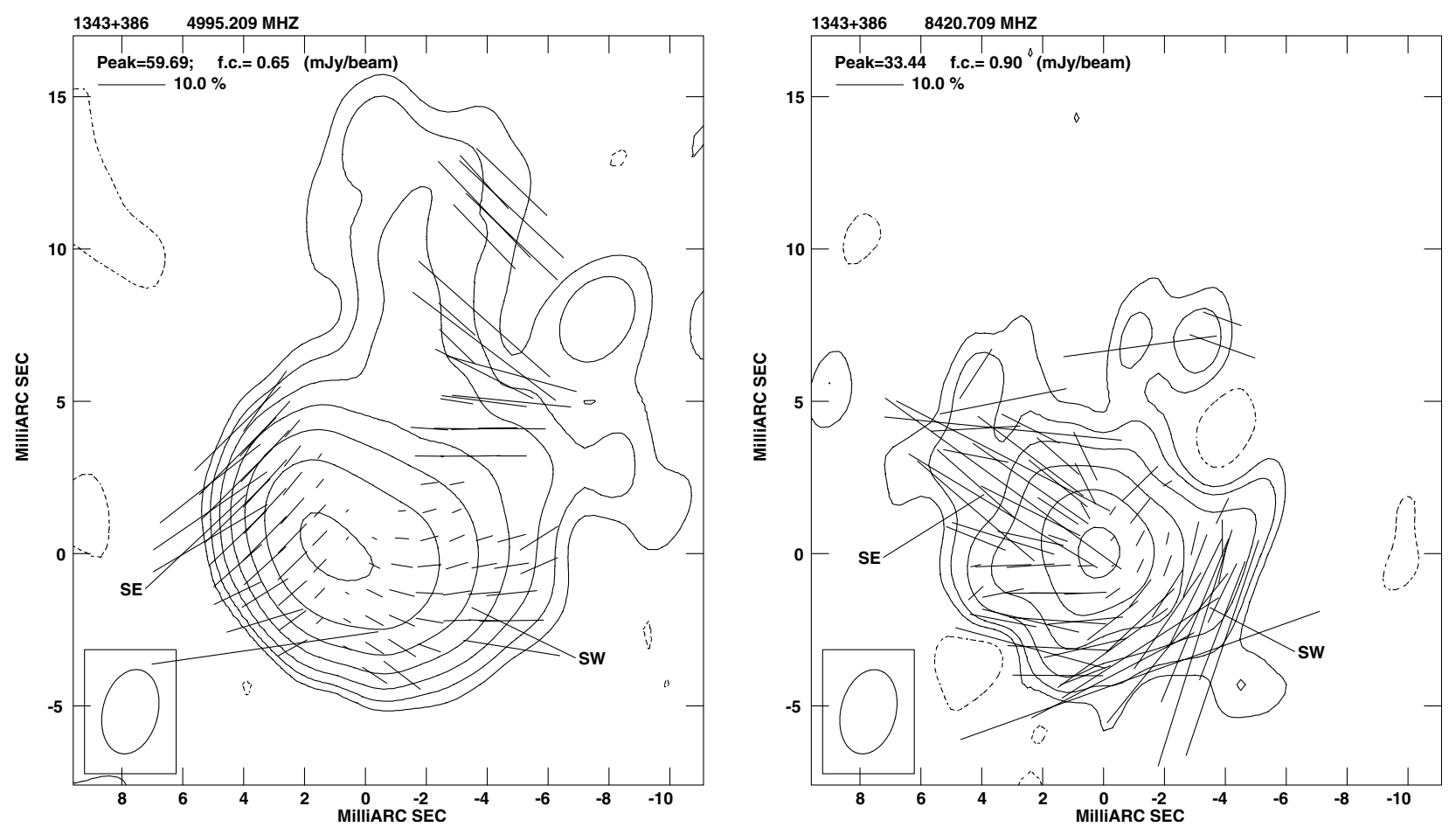

Fig. 4. Polarization images of the Southern lobe of the source B3-1343+386 at $5.0 \mathrm{GHz}($ left) and $8.4 \mathrm{GHz}$ (right). The vectors superimposed on the $I$ contours show the percentage polarization and the position angle of the $\boldsymbol{E}$ vector. The restoring beam is given by the ellipse in the bottom left corner of each image. The image at $8.4 \mathrm{GHz}$ was produced using maxima $u v$-ranges and restoring beam identical to those at $5.0 \mathrm{GHz}$.

B3-2348+450) have a more uncertain morphology, although it is not excluded that they may be CSOs as well.

\subsection{Spectral properties and source growth}

For the 10 sources with three-frequency images, we have studied how the spectral index changes with frequency, and this has proven very effective in establishing the nature of each component (see e.g. B3-0147+400 and B3-2358+406, Fig. 6). Searching for spectral breaks is also important since it provides an estimate of the age of relativistic electrons measured since their last acceleration. In order to compute the radiative age we assumed the equipartition magnetic field calculated with standard formulae (Pacholczyk 1970), assuming particle energy equally distributed into electrons and protons, a filling factor of unity, an ellipsoidal geometry, and considering the radio spectrum from $10 \mathrm{MHz}$ up to $100 \mathrm{GHz}$, as in Murgia et al. (1999) and Murgia (2003).

The spectra of compact regions identified with hot spots are well-fitted by power laws with spectral index of $0.4-0.7$, in agreement with the idea that injection of young electrons occurs. On the other hand, the spectra of lobes and extended features, which are the regions where electrons age, are wellfitted by a JP model ${ }^{2}$ (Jaffe \& Perola 1973).

For seven sources where we could isolate lobe emission not contaminated by neither hot spot nor jet emission (see Table 3),

\footnotetext{
2 The JP model assumes that a single injection of electrons occurs, with pitch angles continuously re-isotropized. In this case, all electrons age in the same way.
}

we have fitted a JP model to the three frequency data and obtained the break frequencies reported in Table 3 . We have to stress that, as we miss some low surface brightness emission at the two higher frequencies, it is likely that the break frequency may occur at frequencies slightly lower than those we find, as the diffuse regions we possibly miss are likely to contain the older electrons. Therefore, the radiative ages we derive are probably an underestimate of the true value and have to be taken as an indication.

We obtained ages $\leq 5 \times 10^{3}$ years, implying that these objects are young. Because of electron reacceleration that takes place in the hot spots, we are not able to determine the radiative age in sources dominated by these regions (e.g. B3-0703+468). Given the source size, we computed also the source expansion velocities which are in the range of $0.1-0.4 h^{-1} \mathrm{c}$, in good agreement with the values of proper motions of the hot spots of Compact Symmetric Objects obtained by Polatidis \& Conway (2003). These results are reported in Table 3.

\subsection{Polarization properties}

Among the 18 sources, only the quasar B3-1343+386 and the galaxy B3-2304+377 show some amount of polarized emission at VLBA resolution. This is consistent with the VLA observations (Paper I) since these are the two only sources among those observed in this experiment with some amount of polarized emission on the VLA scale.

The source B3-1343+386 (Fig. 4) shows polarized emission in its Southern component only, accounting for $8.2 \mathrm{mJy}$ at 


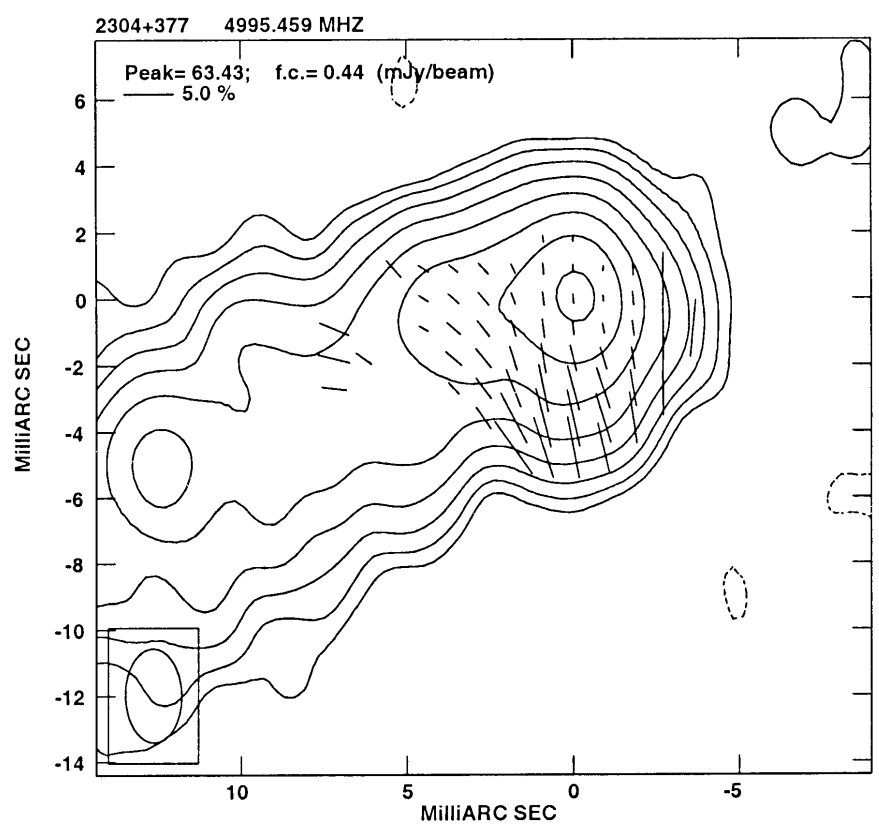

Fig. 5. Polarization image of the Westernmost region of the source B3-2304+377 at 5.0 GHz. The vectors superimposed on the $I$ contours show the percentage polarization and the position angle of the $\boldsymbol{E}$ vector. The restoring beam is given by the ellipse in the bottom left corner.

$8.4 \mathrm{GHz}$ and $6.9 \mathrm{mJy}$ at $5.0 \mathrm{GHz}$, i.e. $6 \%$ and $3 \%$ of the component total flux density (corresponding to $2.5 \%$ and $1.7 \%$ of the source total flux density as measured at the VLA). Since this component is polarized at both frequencies, we are able to compute the Rotation Measure. The polarization angle of the whole component is $\chi \sim 89^{\circ}$ at $8.4 \mathrm{GHz}$ and $\chi \sim 113^{\circ}$ at $5.0 \mathrm{GHz}$, therefore the observed Rotation Measure is $R M \sim 185 \mathrm{rad} \mathrm{m}^{-2}$, if we do not consider any $n \pi$ ambiguity (i.e. $n=0$ ). In this component we can identify two regions (labelled SE and SW in Fig. 4) with very different polarization angles: the SE region has $\chi \sim 75^{\circ}$ at $8.4 \mathrm{GHz}$ and $\sim 134^{\circ}$ at $5.0 \mathrm{GHz}$, while the SW has $\chi \sim 142^{\circ}$ at $8.4 \mathrm{GHz}$ and $\chi \sim 85^{\circ}$ at $5.0 \mathrm{GHz}$. The observed Rotation Measures we obtain are $\sim 450 \mathrm{rad} \mathrm{m}^{-2}$ in SE and $\sim-450 \mathrm{rad} \mathrm{m}^{-2}$ in SW components.

The source B3-2304+377 (Fig. 5) shows polarized emission in the brightest region of its Western component, accounting for $5.1 \mathrm{mJy}$ at $5.0 \mathrm{GHz}$, i.e. $1.5 \%$ of the component total flux density (that is $0.8 \%$ of the source total flux density as measured at the VLA). Since this source has data at $5.0 \mathrm{GHz}$ only, we are not able to estimate the Rotation Measure.

In all the remaining sources no polarization is detected at either frequency at the $3 \sigma$ noise level ( 1 contour level in Figs. 1 and 2). This turns out in very low fractional polarization limits on the brightest features (hot spots), typically less than $0.5-1 \%$ depending on the peak brightness; the upper limit on the low surface brightest regions increases to $10-20 \%$. If the very low level of polarization is due to an external Faraday screen (see Fanti et al. 2004, Paper IV, for a Faraday screen model, and also Cotton et al. 2003) the screen coherence length is required to be smaller than a few parsecs.
The large fractional polarization of the hot spots of B3-1343+386 and B3-2304+377 appears rather surprising when compared to the upper limits on the hot spots of the other sources. We may suppose that these two sources are somewhat oriented along the line of sight (for B3-1343+386 this is suggested by the presence of the bright core and the one-sided jet) so that their real size is much larger than the projected one and the hot spots have already emerged from the inner denser region of the Faraday screen.

In general, the vast majority of the sources studied here can be considered unpolarized at 5.0 and $8.4 \mathrm{GHz}$. Further we can infer that it is likely that beam depolarization is not effective.

\section{Summary}

We have presented the results of new VLBA observations at 5.0 and $8.4 \mathrm{GHz}$ of the most compact sources $\left(L A S<0.3^{\prime \prime}\right)$ in the B3-VLA CSS sample from Fanti et al. (2001). The conclusions we can draw from this investigation are:

- About 55\% of the sources has a "Double/Triple type" structure, with linear sizes ranging from $\sim 200 h^{-1} \mathrm{pc}$ to $\sim 1 h^{-1} \mathrm{kpcs}$;

- In seven sources $(\sim 40 \%)$ the radio core is unambiguously detected;

- The fit to the radio spectrum of the various source components shows that hot spots are well-fitted by a power law with an injection spectral index $\sim 0.4$, while lobes show a significant steepening at high frequencies, well-fitted by the JP model, as expected from synchrotron electron ageing;

- From the study of the integrated spectra we conclude that radiative ages of lobe-dominated CSS sources are $<5 \times 10^{3}$ years, if equipartition magnetic fields are assumed. The corresponding source expansion velocities are in the range of $0.1 h^{-1}$ to $0.4 h^{-1} \mathrm{c}$;

- Only 2 sources are polarized, with values of their percentage polarization $\sim 2.5 \%$ and $<2 \%$ at 8.4 and $5.0 \mathrm{GHz}$ respectively.

Acknowledgements. We are grateful to Dr. M. Murgia for helpful discussions and suggestions on the best use of his software Synage++. The authors acknowledge financial support from the Italian MIUR under grant COFIN-2002-02-8118. The VLBA is operated by the US National Radio Astronomy Observatory which is a facility of the National Science Foundation operated under a cooperative agreement by Associated Universities, Inc. This work has made use of the NASA/IPAC Extragalactic Database NED which is operated by the JPL, California Institute of Technology, under contract with the National Aeronautics and Space Administration. 

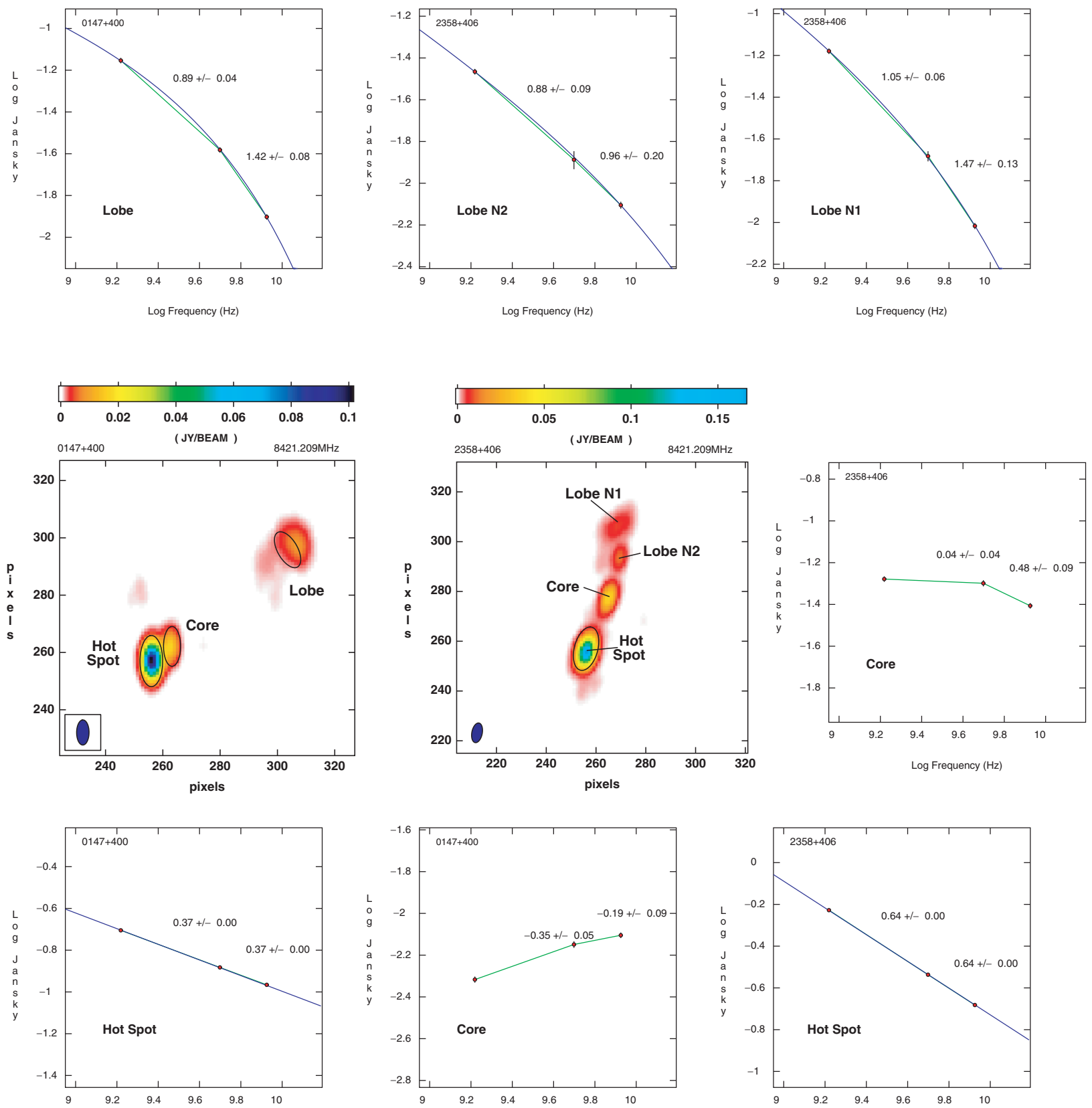

Log Frequency $(\mathrm{Hz})$

Log Frequency $(\mathrm{Hz})$

Log Frequency $(\mathrm{Hz})$

Fig. 6. Examples of spectral fits in the lobes (JP model), hot spot (power law) and core (inverted spectrum) of B3-0147+400 (left) and B3-2358+406 (right). The values of the local spectral indices are reported in the spectrum of each component. The green line connects the flux density measurements, while the blue line represents the best fit to the spectrum. No fit has been attempted for the core region in both sources.

\section{References}

Cotton, W. D., Dallacasa, D., Fanti, C., et al. 2003, PASA, 20, 12

Dallacasa, D., Fanti, C., Fanti, R., Schilizzi, R. T., \& Spencer, R. E. 1995, A\&A, 295, 27

Dallacasa, D., Tinti, S., Fanti, C., et al. 2002a, A\&A, 389, 115 (Paper II)

Dallacasa, D., Fanti, C., Giacintucci, S., et al. 2002b, A\&A, 389, 126 (Paper III)

Fanti, C., Fanti, R., Dallacasa, D., et al. 1995, A\&A, 302, 317 (Paper IV)

Fanti, C., Pozzi, F., Dallacasa, D., et al. 2001, A\&A, 369, 380 (Paper I)

Fanti, C., Branchesi, M., Cotton, W. D., et al. 2004, A\&A, in press

Jaffe, W. J., \& Perola, G. C. 1973, A\&A, 26, 423
Marecki, A., Niezgoda, J., Włodarczak, J., et al. 2003, PASA, 20, 42

Murgia, M., Fanti, C., Fanti, R., et al. 1999, A\&A, 345, 409

Murgia, M. 2003, PASA, 20, 19

Pacholczyk, A. G. 1970, Radio Astrophysics (San Francisco: Freeman \& Co.)

Peck, A. B., \& Taylor, G. B. 2001, ApJ, 554, 147

Polatidis, A. G., \& Conway, J. E. 2003, PASA, 20, 69

Readhead, A.C.S., Taylor, G. B., Xu, W., et al. 1996, ApJ, 460, 612

Snellen, I. A. G., Schilizzi, R. T., Miley, G. K., et al. 2000, MNRAS, 319,445

Snellen, I. A. G., Mack, K. -H., Schilizzi, R. T., \& Tschager, W. 2003, PASA, 20, 38

Vigotti, M., Grueff, G., Perley, R., Clark, B. G., \& Bridle, A. H. 1989, AJ, 98, 419 


\section{Online Material}


M. Orienti et al.: The B3-VLA CSS sample. V., Online Material p 2
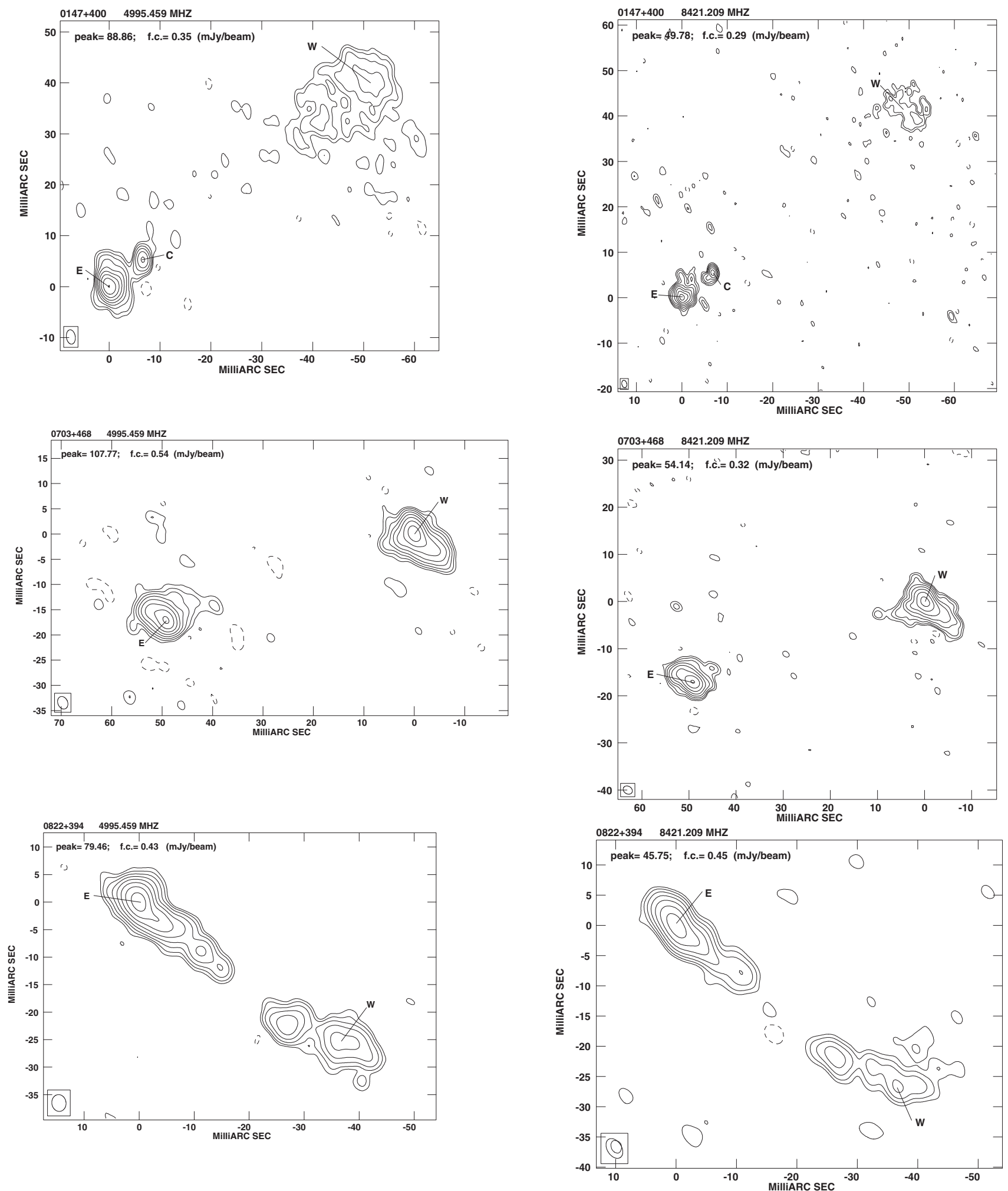

Fig. 1. VLBA images at 5.0 (left) and 8.4 (right) GHz. For each image we give the following information on the plot itself: a) peak flux density in $\mathrm{mJy} /$ beam; b) first contour intensity (f.c., again in mJy/beam), which is generally $=3 \mathrm{rms}$. of the noise; contour levels increase by a factor of 2 ; c) the restoring beam is plotted on the bottom left corner of each image. 
M. Orient et al.: The B3-VLA CSS sample. V., Online Material p 3
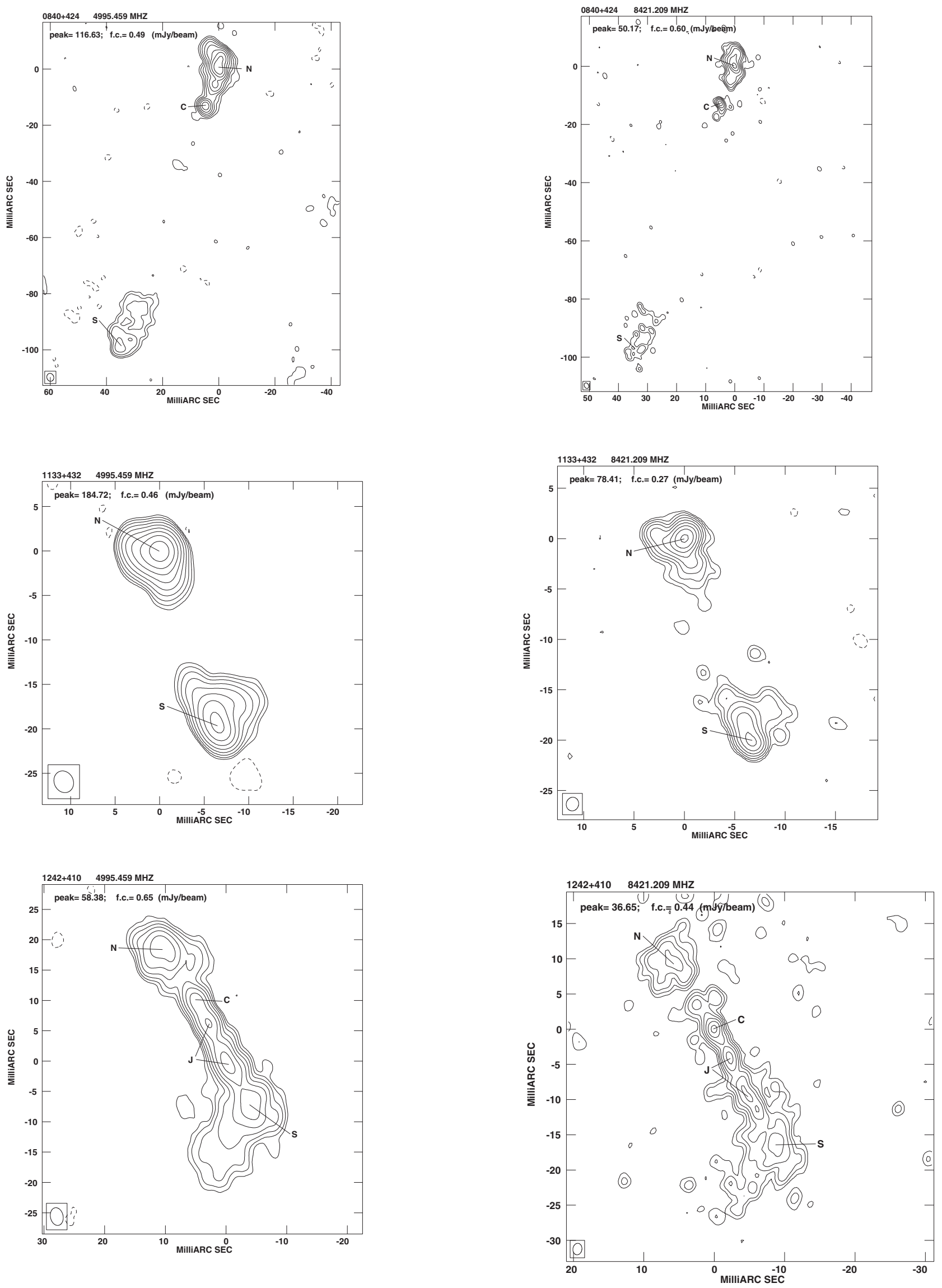

Fig. 1. continued. 
M. Orienti et al.: The B3-VLA CSS sample. V., Online Material p 4
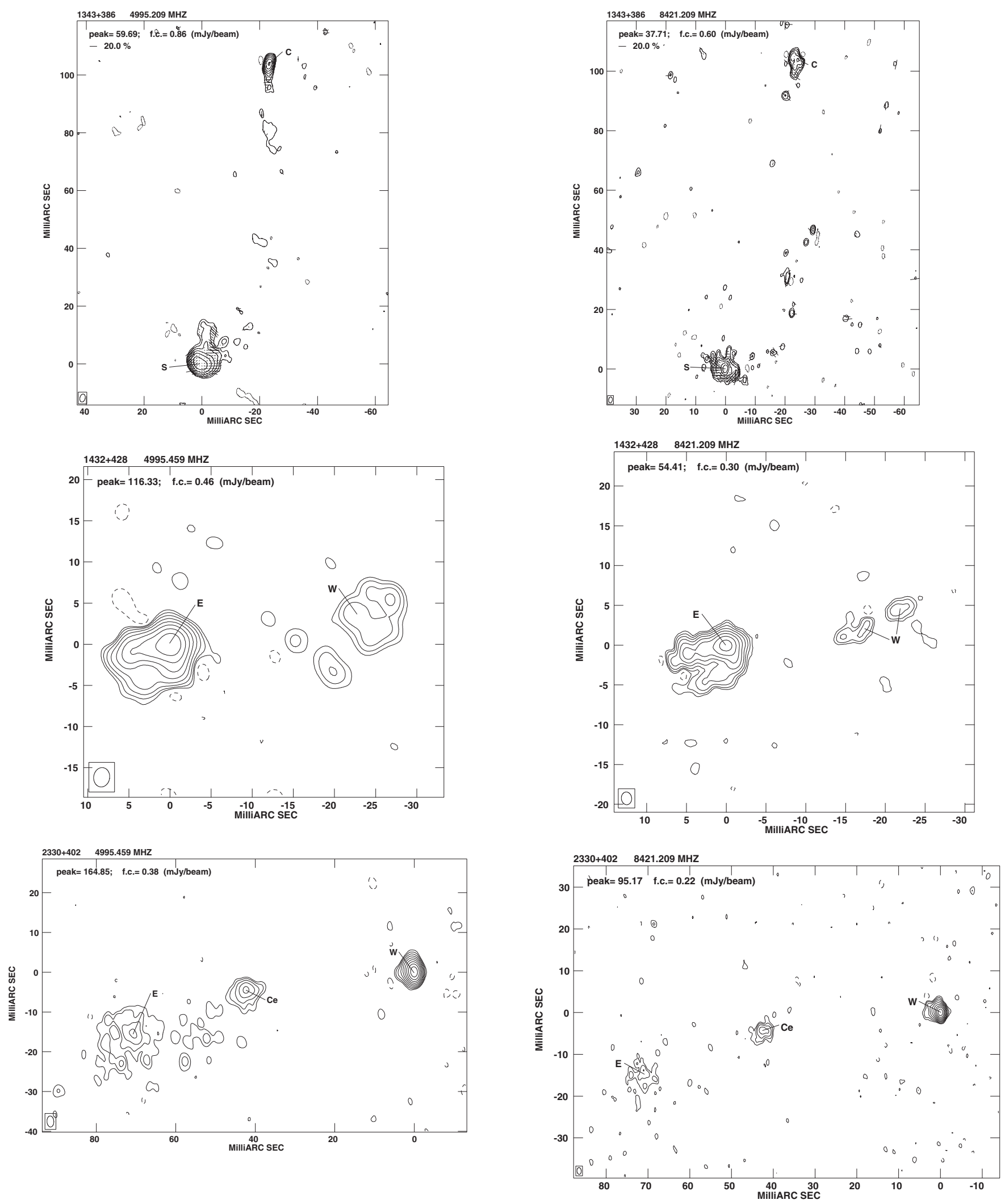

Fig. 1. continued. 
M. Orienti et al.: The B3-VLA CSS sample. V., Online Material p 5
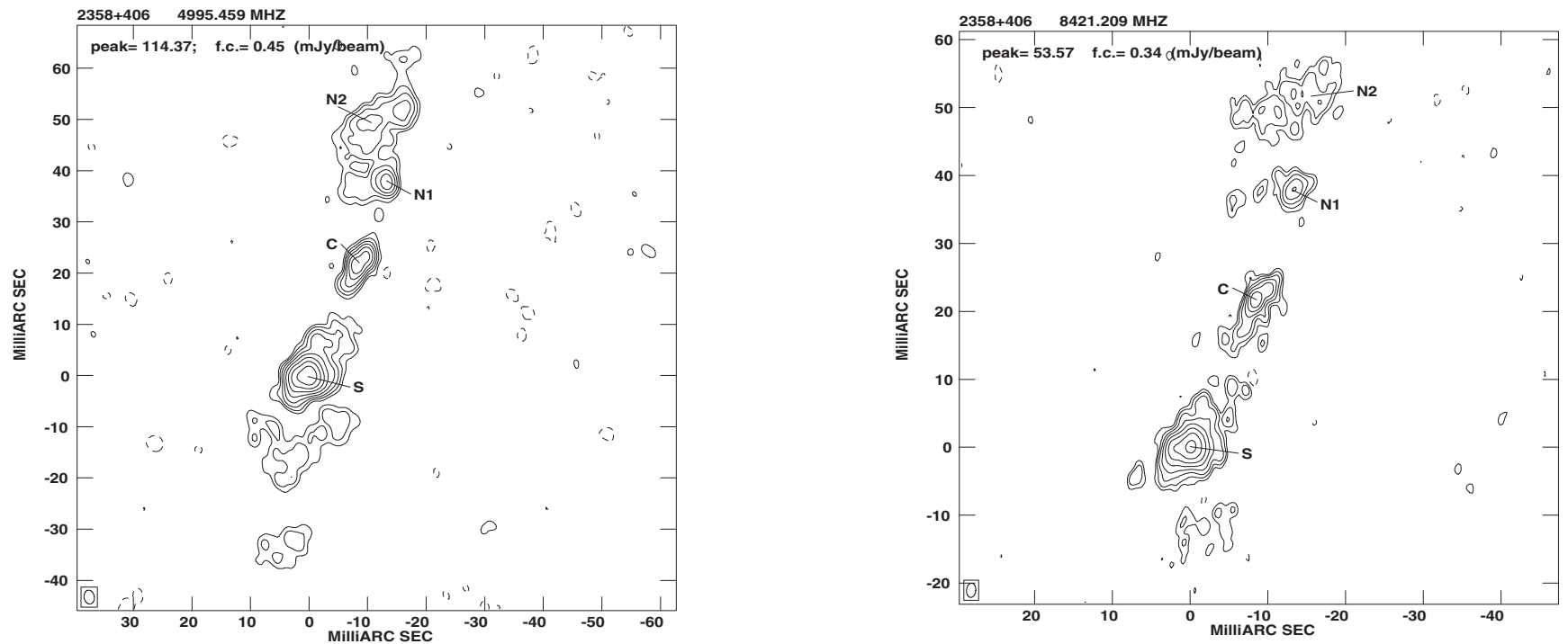

Fig. 1. continued. 
M. Orienti et al.: The B3-VLA CSS sample. V., Online Material p 6
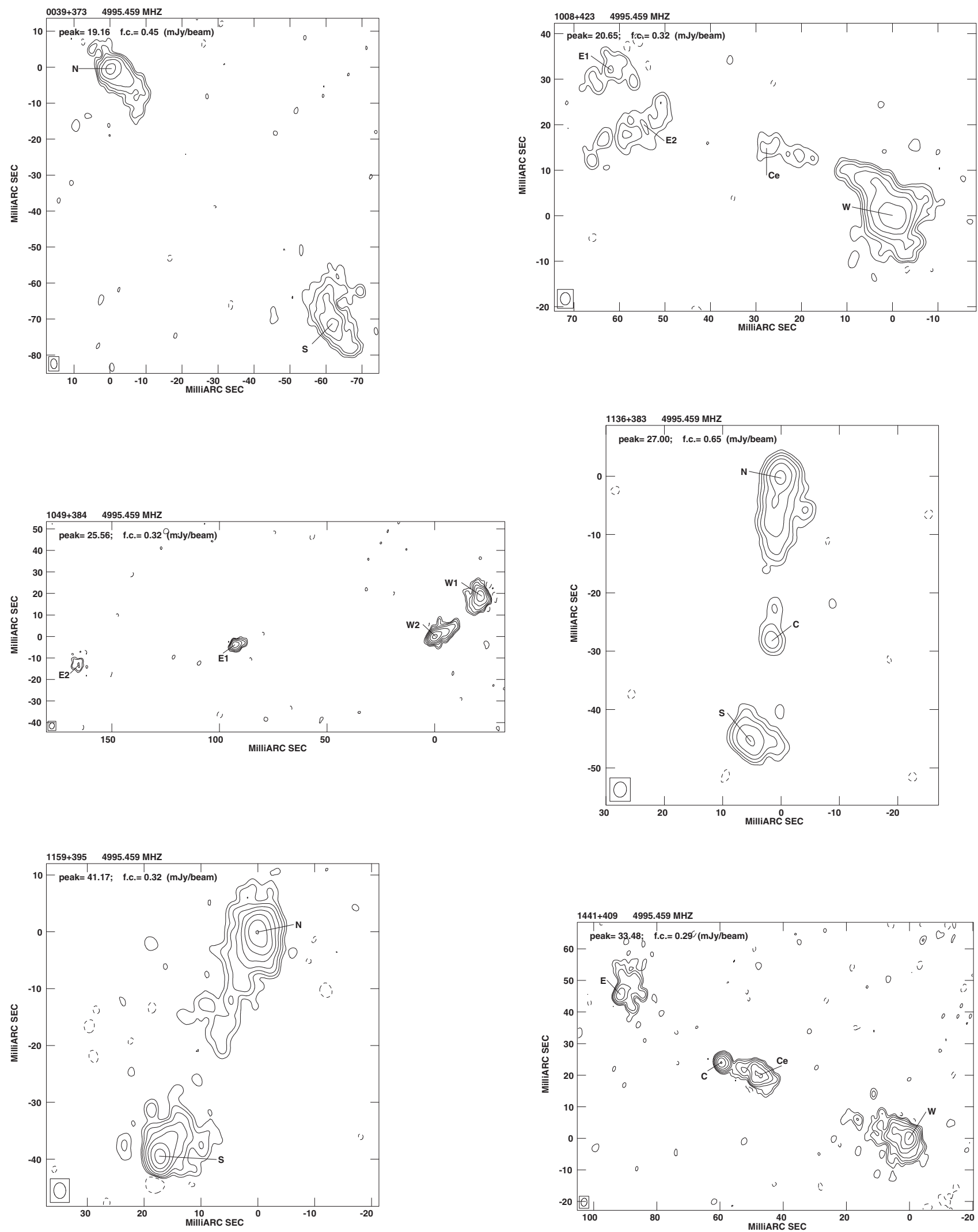

Fig. 2. VLBA images of the sources observed at $5.0 \mathrm{GHz}$ only. For each image we give the following information on the plot itself: a) peak flux density in $\mathrm{mJy} / \mathrm{beam}$; b) first contour intensity (f.c., in mJy/beam), which is generally 3 times the rms. noise on the image plane; contour levels increase by a factor of 2 ; c) the restoring beam is plotted on the bottom left corner of each image. 
M. Orienti et al.: The B3-VLA CSS sample. V., Online Material $p 7$
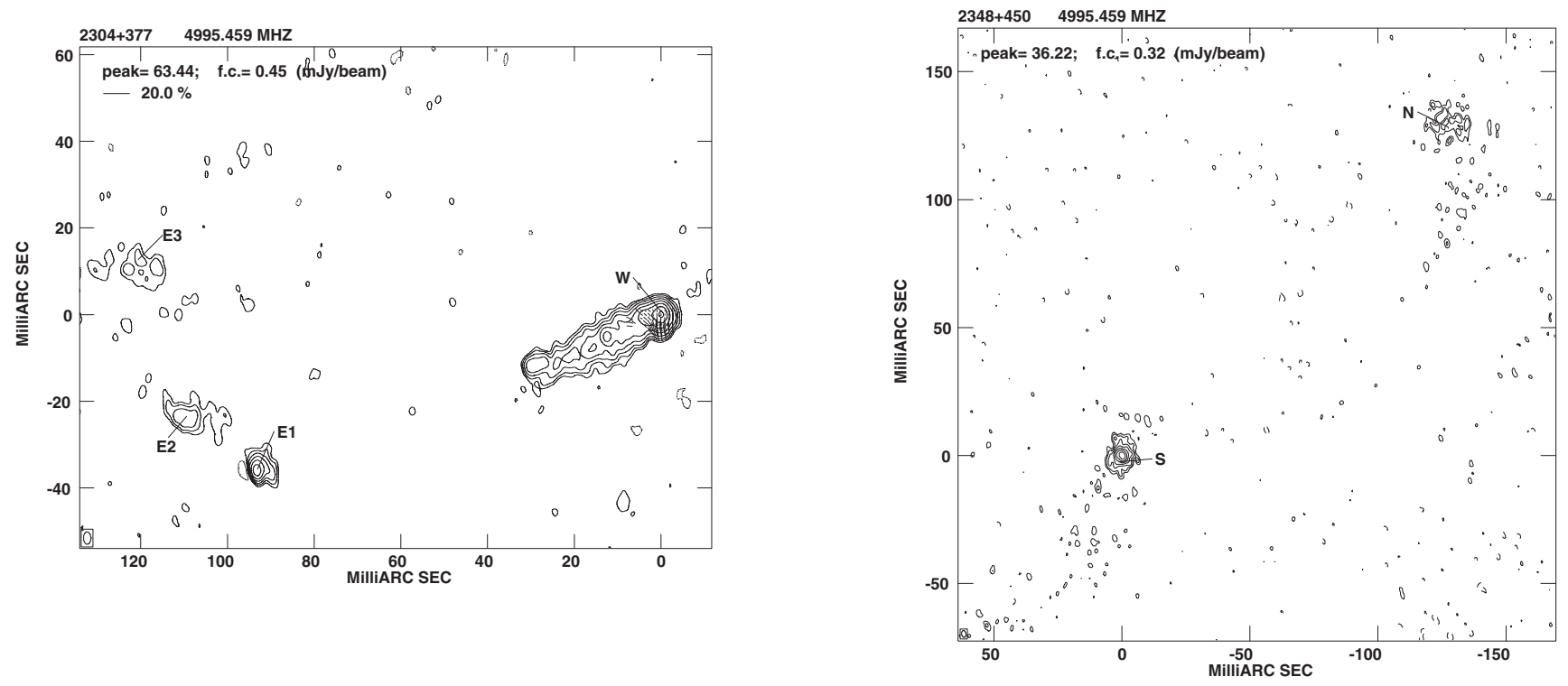

Fig. 2. continued. 

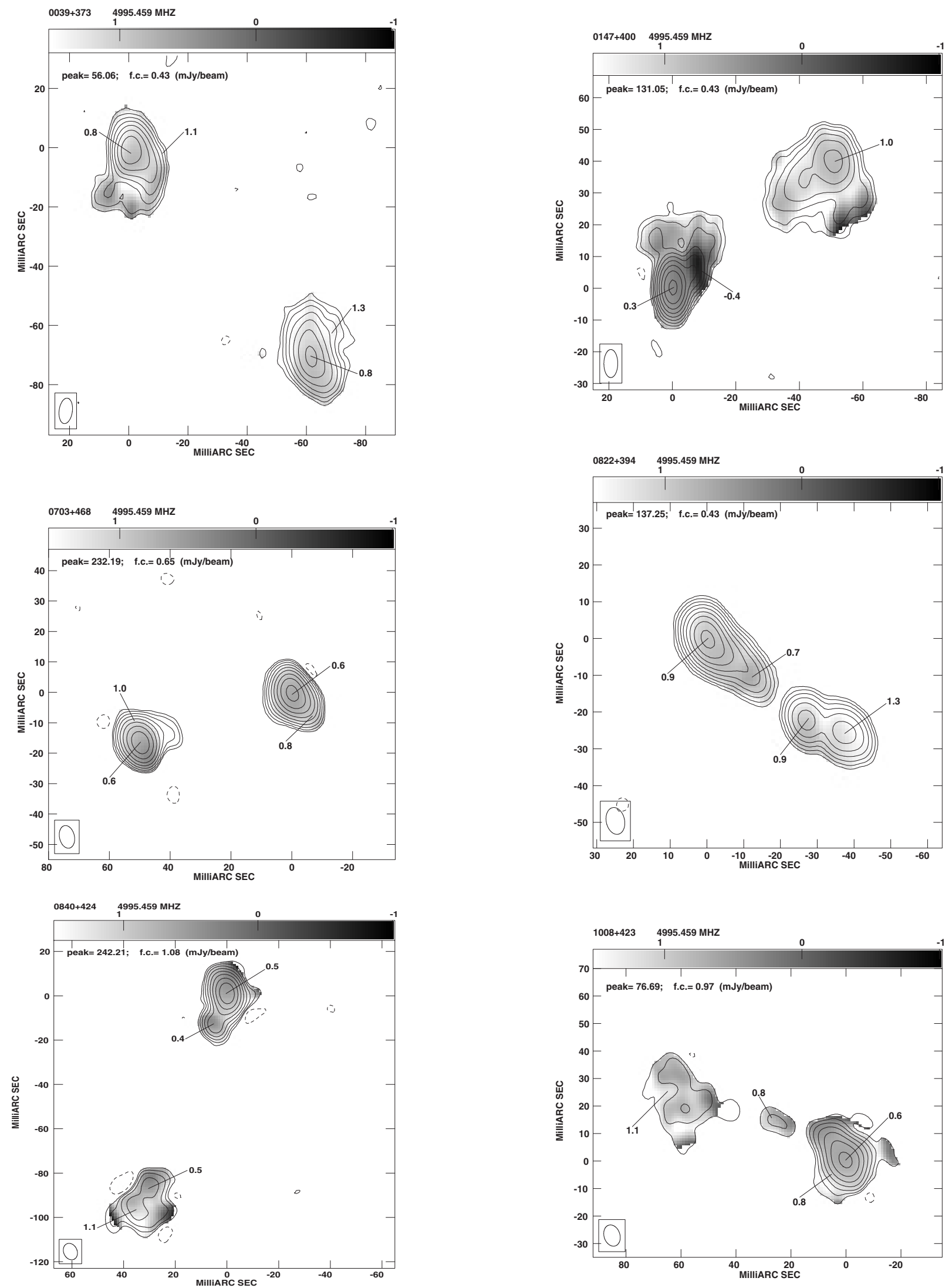

Fig. 3. VLBA spectral images between 1.67 and $5.0 \mathrm{GHz}$. For each spectral index distribution image we provide the following information on the plot itself: a) peak flux density and first contour (f.c.), in mJy/beam at $5 \mathrm{GHz}$, corresponding to 3 times the off-source noise on the image plane; contours increase by a factor 2; b) beam size, represented by the ellipse in the lower left corner; c) grey-scale range of the spectral index $1.5>\alpha>-1$, in the convention $S(v) \propto v^{-\alpha}$. The grey scale is shown by the wedge at the top of each spectral index image. Contours shows the images at $5.0 \mathrm{GHz}$ obtained by using the same uv-range as the $1.67 \mathrm{GHz}$, and the same restoring beam. Local spectral indices are marked. 
M. Orienti et al.: The B3-VLA CSS sample. V., Online Material p 9
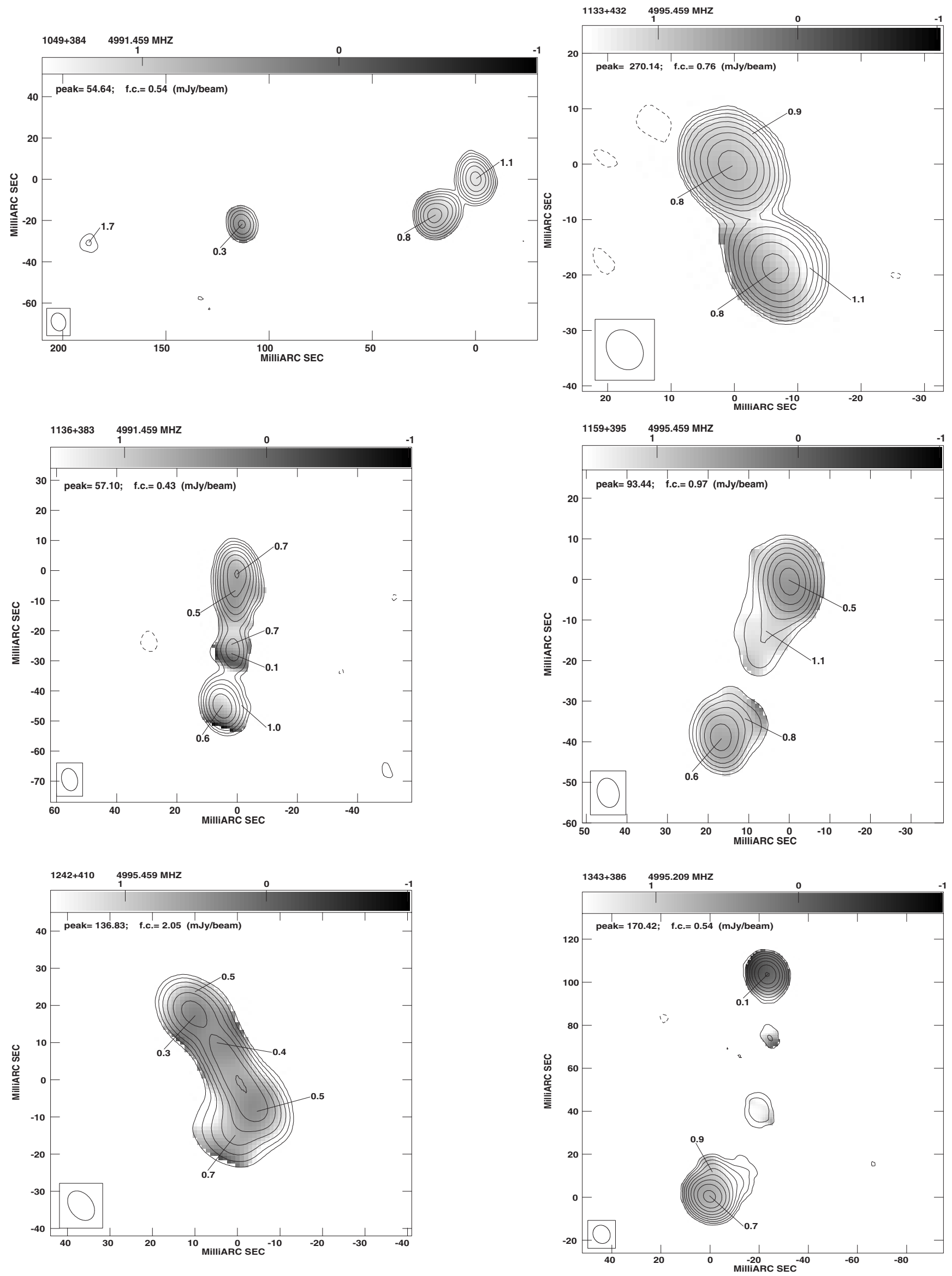

Fig. 3. continued. 
M. Orienti et al.: The B3-VLA CSS sample. V., Online Material p 10
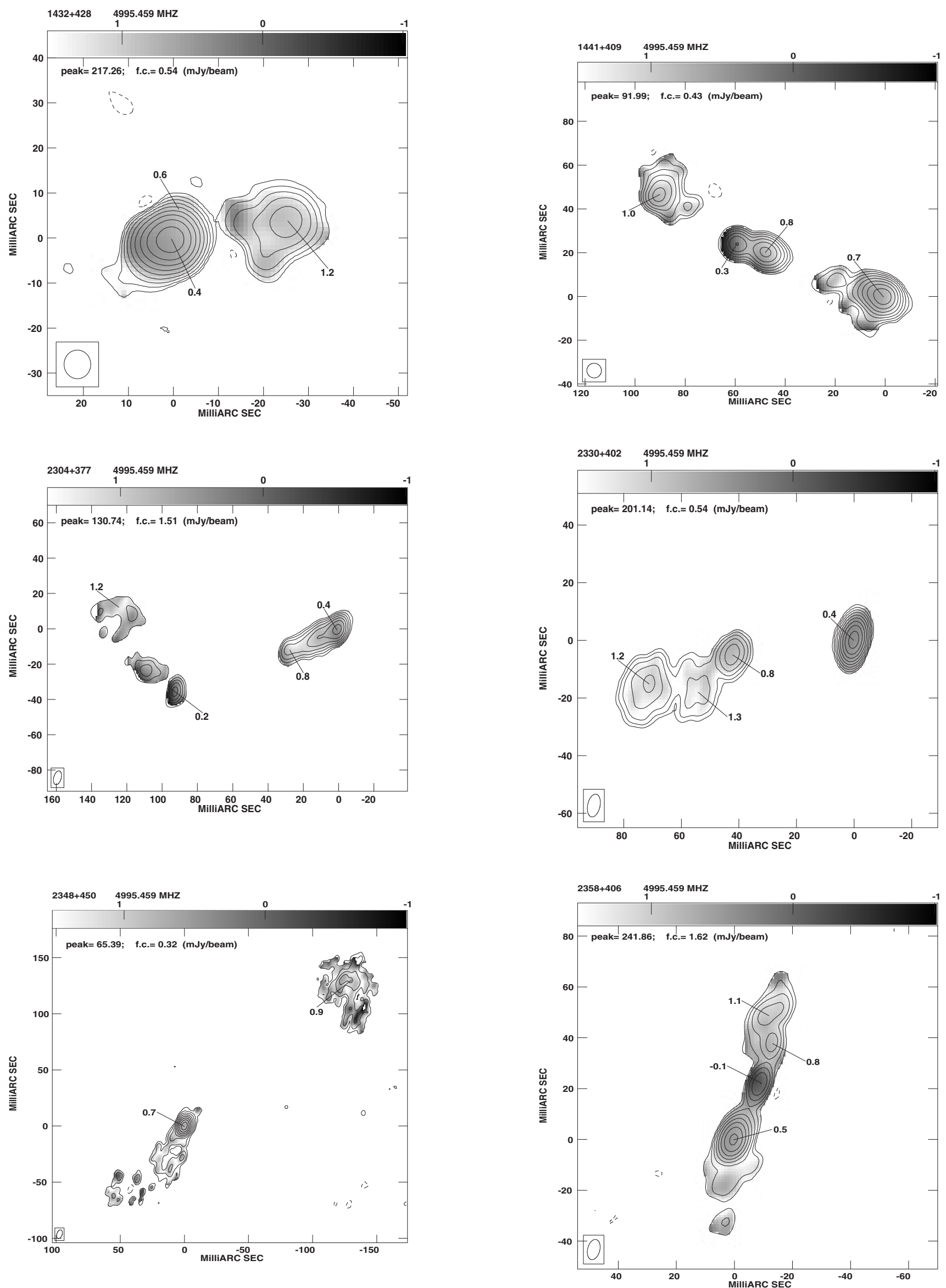

Fig. 3. continued. 